\title{
Effect of Density-of-States Effective Mass on Transport Properties of Two Converging Valence Bands
}

\author{
Hyun-Sik Kim $\mathbb{D}^{\dagger}$ \\ Department of Materials Science and Engineering, Hongik University, Seoul 04066, Korea \\ (Received May 4, 2019; Revised May 13, 2019; Accepted May 14, 2019)
}

\begin{abstract}
Band convergence is known to be effective in improving thermoelectric performance by increasing the Seebeck coefficient without significantly reducing electrical conductivity. Decoupling of the Seebeck coefficient and electrical conductivity in converged bands is the key requirement. Yet, the degree of decoupling depends on the band parameters of the converging bands. Herein, we report theoretical transport properties of two valence bands as their energy difference changes from $0.25 \mathrm{eV}$ to $0 \mathrm{eV}$. In order to demonstrate the effect of band parameters in transport, we first conducted calculations for the case where the two bands have the same parameters. Then, we conducted the same calculation by doubling the density-of-states effective mass of one valence band. Given that there are two bands, each band's effective mass was doubled one at a time while the other band's effective mass remained constant. We found that the decoupling was strongest when the bands participating in convergence had the same band parameters.
\end{abstract}

Key words : Band convergence, Thermoelectric performance, Seebeck coefficient, Decoupling, Effective mass

\section{Introduction}

$\mathrm{T}$ he farthest man-made object from Earth (the spacecraft Voyager 1) runs on radioisotope thermoelectric generators. ${ }^{1)}$ The radioactive radiation (heat) is converted to electricity by the thermoelectric materials surrounding the radioisotope heat source. This reliable material can also convert an applied electrical potential to a temperature gradient (for cooling application). The efficiency of the conversion (from $\Delta T$ to $\Delta V$, and from $\Delta V$ to $\Delta T$ ) is characterized by a dimensionless figure-of-merit $z T=\left(S^{2} \times \sigma\right) / \kappa \times T$, where $S$, $\sigma, \kappa$, and $T$ are the Seebeck coefficient, electrical conductivity, thermal conductivity, and the temperature in Kelvin, respectively. At a glance, creating a high-performance thermoelectric material does not seem too demanding: the material should have a high $S$ and $\sigma$, and a low $\kappa$. However, raising $z T$ becomes non-trivial because of the interconnections among $S, \sigma$, and $\kappa$.

One of the most popular routes to a high $z T$ value is to suppress the thermal conductivity contributed by lattice vibrations $(\kappa){ }^{2)}$ Because $\kappa_{l}$ is not correlated to any other parameters in $z T$, reducing $\kappa_{l}$ is the most straightforward way to improve performance. $z T$ can also be improved by increasing $S$ and $\sigma$ simultaneously. Typically, $S$ is inversely proportional to $\sigma$. For example, when the carrier concentra-

Corresponding author : Hyun-Sik Kim

E-mail : hyunsik.kim@hongik.ac.kr

Tel : +82-2-320-1666 Fax : +82-2-320-1666

ORCID

https://orcid.org/0000-0001-8934-4042 tion of a thermoelectric material is decreased, $S$ increases while $\sigma$ decreases. The density-of-states effective mass $\left(\mathrm{m}^{*}\right)$ of a material is closely related to $S$. The heavier the $m^{*}$, the higher the $S$. Since the mobility of charged particles (electrons or holes) is inversely proportional to $m^{*}$, materials with high $m^{*}$ (high $S$ ) have low $\sigma$. Here, $m^{*}=N_{v}{ }^{2 / 3} \times m_{b}{ }^{*}$, where $N_{v}$ and $m_{b}{ }^{*}$ are the valley degeneracy and the band mass of a single valley, respectively. ${ }^{3)}$ To be more specific, $m_{b}{ }^{*}$ is inversely proportional to the mobility. Therefore, if it is possible to increase $m^{*}$ without increasing $m_{b}{ }^{*}$ (i.e., by increasing $N_{v}$ ), then $S$ can be improved without deteriorating $\sigma$. A simultaneous increase in $S$ and $\sigma$ can be acquired by converging nearby bands (in terms of energy). When two bands converge, the effective $m^{*}$ is increased because of the higher $N_{v}$. Recently, the highest room temperature $z T$ in the $\left(\mathrm{Bi}_{0.25} \mathrm{Sb}_{075}\right)_{2} \mathrm{Te}_{3}$ alloy was explained by two valence bands converging at the $\left(\mathrm{Bi}_{0.25} \mathrm{Sb}_{075}\right)_{2} \mathrm{Te}_{3}$ composition. ${ }^{4}$

Although we now know that converged bands are much better than single band participation in transport, the manner in which the band parameters of the converging bands affect the resulting transport properties is unknown. In this study, we perform two-band model calculations where two valence bands with the same band parameters converge from an energy difference of $0.25 \mathrm{eV}$ to $0 \mathrm{eV}$. Then, we intentionally double $m^{*}$ of one of the bands and repeat the same two-band model calculations. Since two bands exist, we increase $m^{*}$ one at a time while keeping the other band's $m$ * constant. When converged, the case where both bands have the same parameters shows a much better power factor $\left(S^{2}\right.$ $\times \sigma$ ) than that where one of the bands has twice as heavy $m^{*}$ as the other. 


\section{Experimental Procedure}

A two-band model, which is an extension of the single parabolic model, was employed to calculate the resulting transport properties of two converging valence bands.5) Band structures that were postulated in the two-band calculations are described in Fig. 1. Three cases were considered to examine the effect of $m^{*}$ on the transport of the two converging bands. Firstly, we considered the band parameters $m^{*}$ and deformation potential $\left(E_{d e f}\right)$ of the two participating valence bands $\left(\mathrm{VB}_{1}\right.$ and $\mathrm{VB}_{2}$ in Fig. 1) to be equal, as shown in Fig. 1(a) (Case I). The energy difference $(\Delta E)$ between the upper valence band $\left(\mathrm{VB}_{1}\right)$ and the lower valence band $\left(\mathrm{VB}_{2}\right)$ was varied from $0.25 \mathrm{eV}$ to $0 \mathrm{eV}$ (when $\Delta E=0 \mathrm{eV}, \mathrm{VB}_{1}$ and $\mathrm{VB}_{2}$ were converged). Secondly, only $m^{*}$ of $\mathrm{VB}_{2}$ (in Fig. 1(a)) was doubled while keeping the other parameters the same as those in the first case (Fig. 1(b), Case II). Lastly, $m^{*}$ of $\mathrm{VB}_{1}$ (in Fig. 1(a)) was doubled while keeping the other parameters the same as those in the first case (Fig. 1(c), Case III). The valley degeneracies $\left(N_{v}\right)$ of the two valence bands $\left(\mathrm{VB}_{1}\right.$ and $\left.\mathrm{VB}_{2}\right)$ were assumed to be 6 (the same as those of $\left.\mathrm{Bi}_{2} \mathrm{Te}_{3}\right)$. When the two bands were converged $(\Delta E=$ $0 \mathrm{eV}$ ), the total $N_{v}$ of the converged bands increased to approximately 12 .

The energy gap $\left(E_{g}\right)$ between the upper valence band $\left(\mathrm{VB}_{1}\right)$ and the lowest conduction band (CB) was not changed in all three cases. CB illustrated in Fig. 1 was not included in the two-band model calculations. Only $E_{g}$ was taken into consideration to account for the change in $m^{*}$ with the modification in $E_{g}$ (as $E_{g}$ is reduced, $m^{*}$ is known to decrease as well because of the interaction between the highest valence band and the lowest conduction band according to the Kane dispersion relation). ${ }^{6)}$ We utilized the same band gap $(0.16 \mathrm{eV}$, as in Table 1) for all three cases. Using the band structures described in Fig. 1, Boltzmann transport equations were
Table 1. Band Parameters Adopted in the Two-Band Model Calculations

\begin{tabular}{|c|c|c|c|}
\hline Band parameters & Case I & Case II & Case III \\
\hline $\begin{array}{l}\text { Upper Valence Band }\left(\mathrm{VB}_{1}\right) \\
m^{*} \text { (in } \mathrm{m}_{0} \text { ) }\end{array}$ & 0.7 & 0.7 & 1.4 \\
\hline $\begin{array}{l}\text { Upper Valence Band }\left(\mathrm{VB}_{1}\right) \\
E_{\text {def }}(\mathrm{eV})\end{array}$ & & 18.7 & \\
\hline $\begin{array}{l}\text { Upper Valence Band }\left(\mathrm{VB}_{1}\right) \\
\text { Valley degeneracy, } N_{v}\end{array}$ & & 6 & \\
\hline $\begin{array}{l}\text { Lower Valence Band }\left(\mathrm{VB}_{2}\right) \\
m^{*}\left(\text { in } \mathrm{m}_{0}\right)\end{array}$ & 0.7 & 1.4 & 0.7 \\
\hline $\begin{array}{l}\text { Lower Valence Band }\left(\mathrm{VB}_{2}\right) \\
E_{\text {def }}(\mathrm{eV})\end{array}$ & & 18.7 & \\
\hline $\begin{array}{l}\text { Lower Valence Band }\left(\mathrm{VB}_{1}\right) \\
\text { Valley degeneracy, } N_{v}\end{array}$ & & 6 & \\
\hline $\begin{array}{l}\text { Band gap } \\
E_{g}(\mathrm{eV})\end{array}$ & & 0.16 & \\
\hline $\begin{array}{l}\text { Energy difference } \\
\text { between } \mathrm{VB}_{1} \text { and } \mathrm{VB}_{2} \\
\Delta E(\mathrm{eV})\end{array}$ & & $0-0.25$ & \\
\hline
\end{tabular}

computationally solved as a function of chemical potential for each valence band $\left(\mathrm{VB}_{1}\right.$ and $\left.\mathrm{VB}_{2}\right)$. Conductivity-weighted averages of the calculated thermoelectric parameters (Seebeck coefficient, electrical conductivity, power factor, mobility, and carrier concentration) of individual bands were computed to acquire the combined transport properties of the two converging valence bands.

The band parameters employed in the two-band model calculations are listed in the Table $1 . E_{g}$ and the band parameters of $\mathrm{VB}_{1}$ in Case I (Fig. 1(a)) are similar to those of $\mathrm{Bi}_{2} \mathrm{Te}_{3}$. However, $\Delta E$ and the parameters for $\mathrm{VB}_{2}$ in Case II $\left(\mathrm{VB}_{1}\right.$ in Case III) are artificial. These values were carefully selected to efficiently illustrate the effect of $m^{*}$ in the band convergence model calculation. (a) Case I

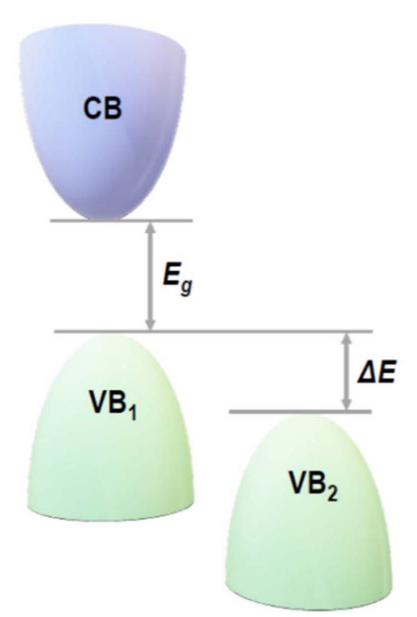

(b) Case II
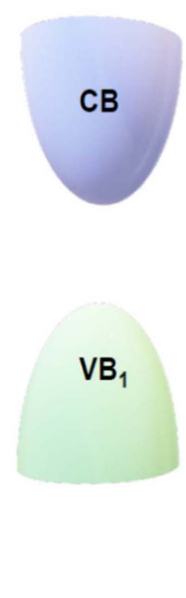

(c) Case III
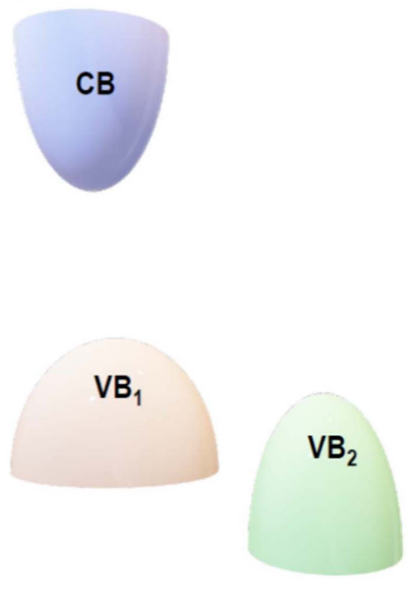

Fig. 1. Schematic diagrams of band structures used in the two-band calculations: (a) Case I (equal $m^{*}$ ), (b) Case II (lower valence band $m^{*}$ doubled), (c) Case III (upper valence band $m^{*}$ doubled). 


\section{Results and Discussion}

Band convergence is one of the most powerful strategies to enhance the electrical properties of thermoelectric materials. While the majority of methods that are known to improve electrical transport increase the Seebeck coefficient (or electrical conductivity) significantly with accompanying electrical conductivity (or Seebeck coefficient) reductions to a lesser magnitude, band convergence enhances the Seebeck coefficient and electrical conductivity simultaneously. The simultaneous increase in both the Seebeck coefficient and electrical conductivity is only possible because the number of valley degeneracies $\left(N_{v}\right)$, and not the single band mass $\left(m_{b}{ }^{*}\right)$, are increased in the band convergence. Consequently, even when the total density-of-states effective mas $\left(m^{*}=N_{v}{ }^{2 / 3} \times m_{b}{ }^{*}\right)$ is increased as a result of the convergence (increase in $N_{v}$ ), its mobility (inversely proportional to $m_{b}{ }^{*}$ ), and of course, the electrical conductivity, remain undamaged.

Furthermore, at the convergence, the electrical conductivities of both bands are summed to produce a higher total electrical conductivity. This can be expressed as Eq. (1) below.

$$
\sigma_{\text {total }}=\sigma_{\mathrm{VB}_{1}}+\sigma_{\mathrm{VB}_{2}}
$$

Therefore, we expect the electrical conductivity of the converged bands $\left(\sigma_{\text {total }}\right)$ to be larger than the electrical conductivity contributions of the individual bands $\left(\sigma_{\mathrm{VB} 1}\right.$ or $\left.\sigma_{\mathrm{VB} 2}\right)$. Here, the electrical conductivity of an individual band can be calculated using Eq. (2), under the single parabolic band (SPB) model, with acoustic phonon scattering being dominant.

$$
\sigma_{\mathrm{SPB}}=\frac{\mu_{H}}{R_{H}}
$$

where

$$
\mu_{H}=\frac{3 \pi \hbar^{4} v_{l}^{2} d}{\sqrt{2} E_{\mathrm{def}}^{2} m^{* 5 / 2}(k T)^{3 / 2}} \frac{F_{-1 / 2}(\eta)}{2 F_{0}(\eta)}
$$

and

$$
R_{H}=\frac{3 h^{3}}{3 \pi\left(2 m^{*} k T\right)^{3 / 2}} \frac{F_{-1 / 2}(\eta)}{2 F_{0}^{2}(\eta)}
$$

$\mu_{H}$ and $R_{H}$ in Eq. (2) are the Hall mobility and Hall coefficient, respectively. $e, v_{l}, d$, and $k$ in Eqs. (3) and (4) are the electric charge, average phonon velocity, density, and the Boltzmann constant, respectively. Lastly, $F_{j}(\eta)$ is the Fermi integral, as detailed below ( $\eta$ is the reduced chemical potential).
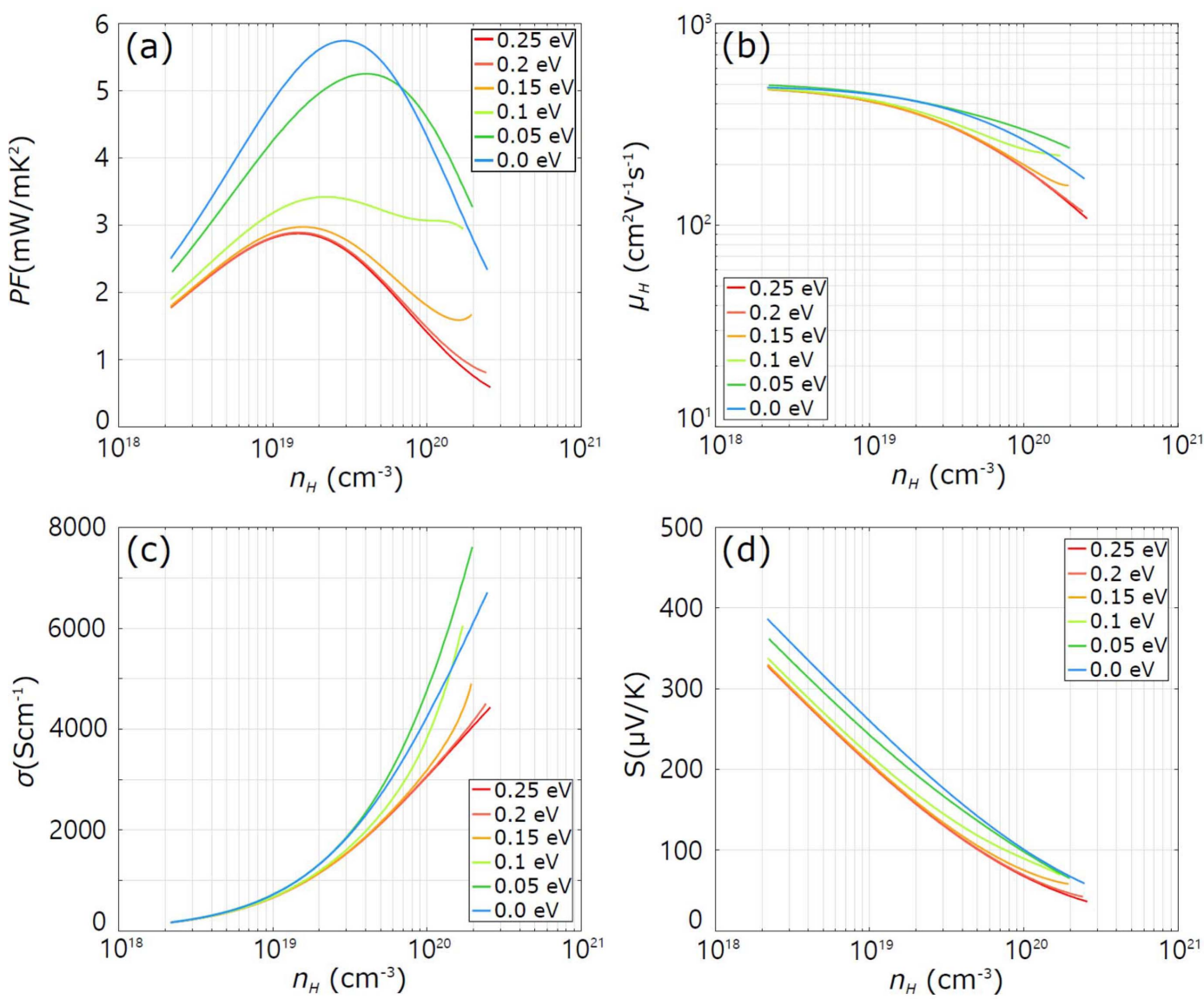

Fig. 2. Calculated transport properties of Case I: (a) power factor, (b) Hall mobility, (c) electrical conductivity, and (d) Seebeck coefficient as a function of Hall carrier concentration. 


$$
F_{j}(\eta)=\int_{0}^{\infty} \frac{\varepsilon^{j} d \varepsilon}{1+\exp (\varepsilon-\eta)}
$$

The total Seebeck coefficient of the converged bands $\left(S_{\text {total }}\right)$ can be expressed as a conductivity-weighted average of the Seebeck coefficient contributions of the individual bands $\left(S_{\mathrm{VB} 1}\right.$ or $S_{\mathrm{VB} 2}$ ), as in Eq. (6). Again, the Seebeck coefficient of an individual band can be calculated using Eq. (7), under the SPB model, with acoustic phonon scattering being dominant.

$$
\begin{aligned}
& S_{\text {total }}=\left(\sigma_{\mathrm{VB}_{1}} S_{\mathrm{VB}_{1}}+\sigma_{\mathrm{VB}_{2}} S_{\mathrm{VB}_{2}}\right) /\left(\sigma_{\mathrm{VB}_{1}}+\sigma_{\mathrm{VB}_{2}}\right) \\
& S_{\mathrm{SPB}}=\frac{k}{e}\left(\frac{2 F_{1}(\eta)}{F_{0}(\eta)}+\eta\right)
\end{aligned}
$$

We first carried out the two-band model calculations for the two valence bands with equal band parameters (density-of-states effective mass, deformation potential, and number of valley degeneracies). For the upper valence band $\left(\mathrm{VB}_{1}\right.$ in Fig. 1(a)), which is fixed in terms of energy, the position of the lower valence band $\left(\mathrm{VB}_{2}\right.$ in Fig. $\left.1(\mathrm{~b})\right)$ with respect to $\mathrm{VB}_{1}$ was varied from $0.25 \mathrm{eV}$ to $0 \mathrm{eV}$. When the energy difference between $\mathrm{VB}_{1}$ and $\mathrm{VB}_{2}(\Delta E)$ is $0.25 \mathrm{eV}$, the location of $\mathrm{VB}_{2}$ is $0.25 \mathrm{eV}$ below $\mathrm{VB}_{1}$ in terms of energy. When $\Delta E$ is $0 \mathrm{eV}$, the two bands $\left(\mathrm{VB}_{1}\right.$ and $\left.\mathrm{VB}_{2}\right)$ converge. More specifi- cally, $\mathrm{VB}_{2}$ now has the same energy as that of the fixed $\mathrm{VB}_{1}$. According to Fig. 2(d), the Seebeck coefficient of the converged bands ( $0.0 \mathrm{eV}$, blue solid line) is the highest, as we expected. It is interesting to note that when $\Delta E=0.1 \mathrm{eV}$ (yellow-green solid line), the lower valence band $\left(\mathrm{VB}_{2}\right)$ begins influencing the transport properties. In other words, if the lower valence band is more than $0.1 \mathrm{eV}$ apart from the highest valence band, that band will not participate seriously in transport (this can be changed if the parameters of the bands are altered). Except for the $0.0 \mathrm{eV}$ line (converged blue solid line), the gradients of the other lines for $\Delta E>0 \mathrm{eV}$ were changed at higher Hall carrier concentrations $\left(n_{H}\right)$. When $\Delta E$ is the largest $(0.25 \mathrm{eV}$, red solid line), the slope of the line flattens at $n_{H}$ values exceeding $2 \mathrm{E} 20\left(\mathrm{~cm}^{-3}\right)$. On the contrary, if $\Delta E$ is only $0.05 \mathrm{eV}$ (green solid line), the change in the slope starts early at $2 \mathrm{E} 19\left(\mathrm{~cm}^{-3}\right)$. A larger $\Delta E$ means that the lower valence band $\left(\mathrm{VB}_{2}\right)$ is far from the upper valence band $\left(\mathrm{VB}_{1}\right)$. In that case, the chemical potential corresponding to the tip of the lower valence band $\left(\mathrm{VB}_{2}\right)$ is much larger than that corresponding to the tip of the upper valence band $\left(\mathrm{VB}_{1}\right)$. Therefore, when we sweep through the band structure with varying chemical potentials, if $\Delta E$ is large, increasing the chemical potential to reach the tip of $\mathrm{VB}_{2}$ will take a long time. Since the Hall carrier concentration $\left(n_{H}\right)$ is directly proportional to the chemical potential,
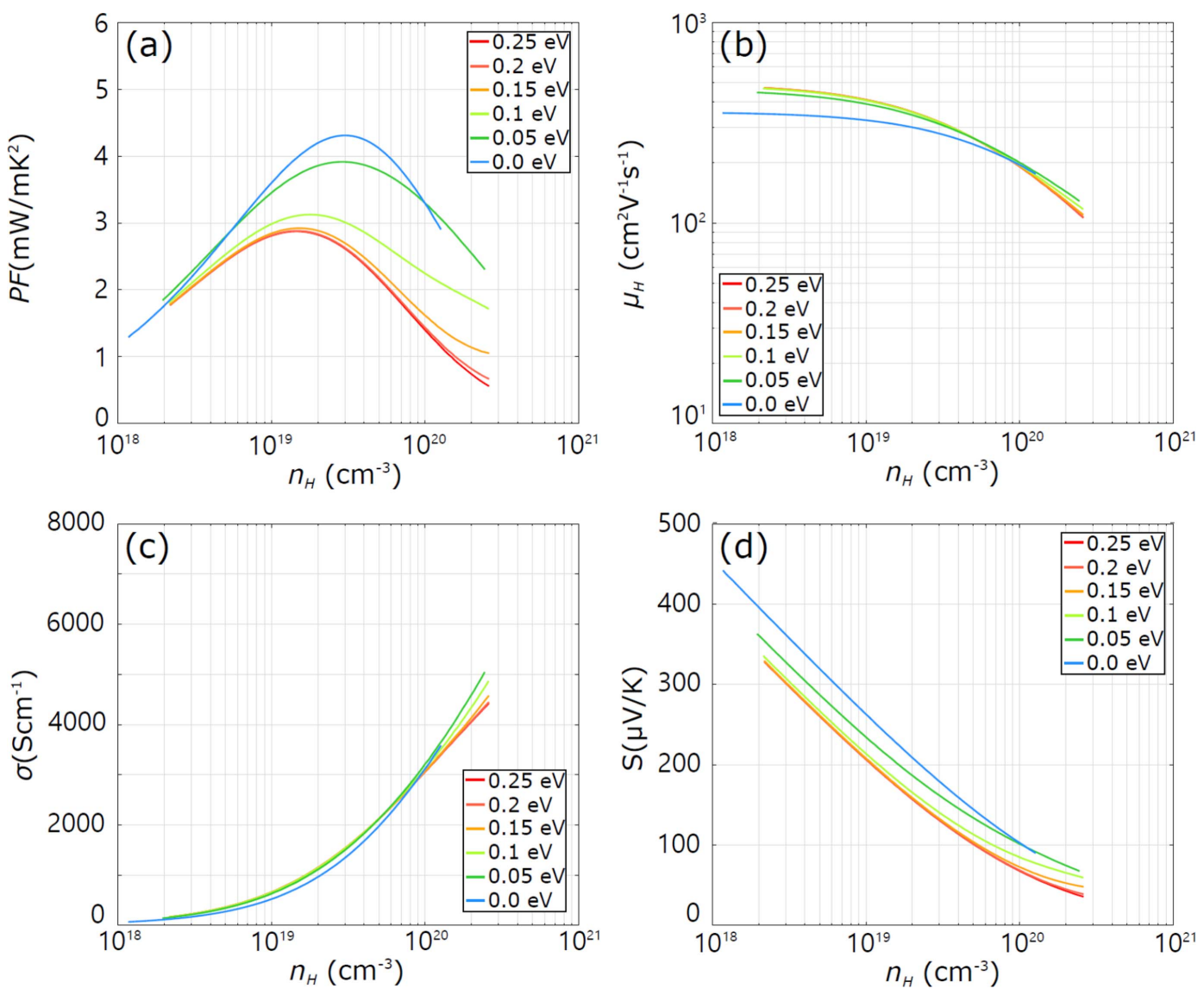

Fig. 3. Calculated transport properties of Case II: (a) power factor, (b) Hall mobility, (c) electrical conductivity, and (d) Seebeck coefficient as a function of Hall carrier concentration. 
the change of the slope in the Seebeck Pisarenko relation (Fig. 2(d)) indicates the instant when the chemical potential encounters the tip of $\mathrm{VB}_{2}$. When $\Delta E=0.05 \mathrm{eV}$ (green solid line in Fig. 2(d)), $\mathrm{VB}_{2}$ starts contributing to the transport from $n_{H} \sim 2 \mathrm{E} 19\left(\mathrm{~cm}^{-3}\right)$, and when $\Delta E=0.25 \mathrm{eV}$ (red solid line in Fig. 2(d)), $\mathrm{VB}_{2}$ starts contributing to the transport only at $n_{H} \sim 2 \mathrm{E} 20\left(\mathrm{~cm}^{-3}\right)$. In both cases, the lines flatten (the rate of the Seebeck coefficient decrease with increasing $n_{H}$ slows down) with the advent of the lower valence band $\left(\mathrm{VB}_{2}\right)$.

The Hall mobilities of the converging valence bands were also calculated (Fig. 2(b)). Analogically, when bands are converged, we can think of it as increasing the number of lanes on a highway along which charged particles (holes or electrons) are travelling. Therefore, the mobility of the holes (or electrons) in the converged bands will improve. Indeed, this was observed in the calculated Hall mobilities $\left(\mu_{H}\right)$ of the two converging bands with the same parameters. According to Eq. (1) above, the total electrical conductivity of the converged band $(0.0 \mathrm{eV}$, blue solid line in Fig. 2(c)) is much greater than that of the large $\Delta E(0.25 \mathrm{eV}$, red solid line in Fig. 2(c)). At $n_{H}>4 \times 10^{19}\left(\mathrm{~cm}^{-3}\right)$, the electrical conductivity of $\Delta E=0.05 \mathrm{eV}$ (green solid line in Fig. 2(c)) is higher than that of the converged one $(\Delta E=0.0 \mathrm{eV}$, blue solid line in Fig. 2(c)). A similar phenomenon is observed in the $n_{H^{-}}$dependent Hall mobilities graph (Fig. 2(b)). Starting at the same
$n_{H}\left(4 \times 10^{19}\left(\mathrm{~cm}^{-3}\right)\right)$, the position of the Hall mobility for $\Delta E=$ $0.05 \mathrm{eV}$ (green solid line) and that for the converged one (0.0 $\mathrm{eV}$, blue solid line) is reversed. For the converged case (0.0 $\mathrm{eV}$, blue solid line), individual Hall mobilities from each band and the combined Hall mobility from the converged bands are the same. This is because the tips of the two bands have the same chemical potentials. However, for $\Delta E$ $=0.05 \mathrm{eV}$ (green solid line), the chemical potential of $\mathrm{VB}_{1}$ maximum is smaller than that of $\mathrm{VB}_{2}$ maximum (Fig. 1(a)). If we are to compare the converged case and that of $\Delta E=$ $0.05 \mathrm{eV}$, the chemical potential of $\mathrm{VB}_{2}$ maximum for the case of $\Delta E=0.05 \mathrm{eV}$ is equal to those of the $\mathrm{VB}_{1}$ and $\mathrm{VB}_{2}$ maxima of the converged case. Simultaneously, the chemical potential of $\mathrm{VB}_{1}$ maximum for the case of $\Delta E=0.05 \mathrm{eV}$ is the smallest among all the bands in both cases. This shows that the highest individual Hall mobility (as a function of chemical potential) occurs for $\mathrm{VB}_{1}$ with $\Delta E=0.05 \mathrm{eV}$ according to Eq. (3) (the individual Hall mobility from $\mathrm{VB}_{2}$ with $\Delta E$ $=0.05 \mathrm{eV}$ is equal to those of $\mathrm{VB}_{1}$ and $\mathrm{VB}_{2}$ from the converged case). Therefore, the combined Hall mobility (function of chemical potential) for $\Delta E=0.05 \mathrm{eV}$ is greater than that of the converged case at all chemical potentials. When the combined electrical conductivity is plotted as a function of chemical potential, the combined electrical conductivity of the converged case is higher than that of $\Delta E=0.05 \mathrm{eV}$. The
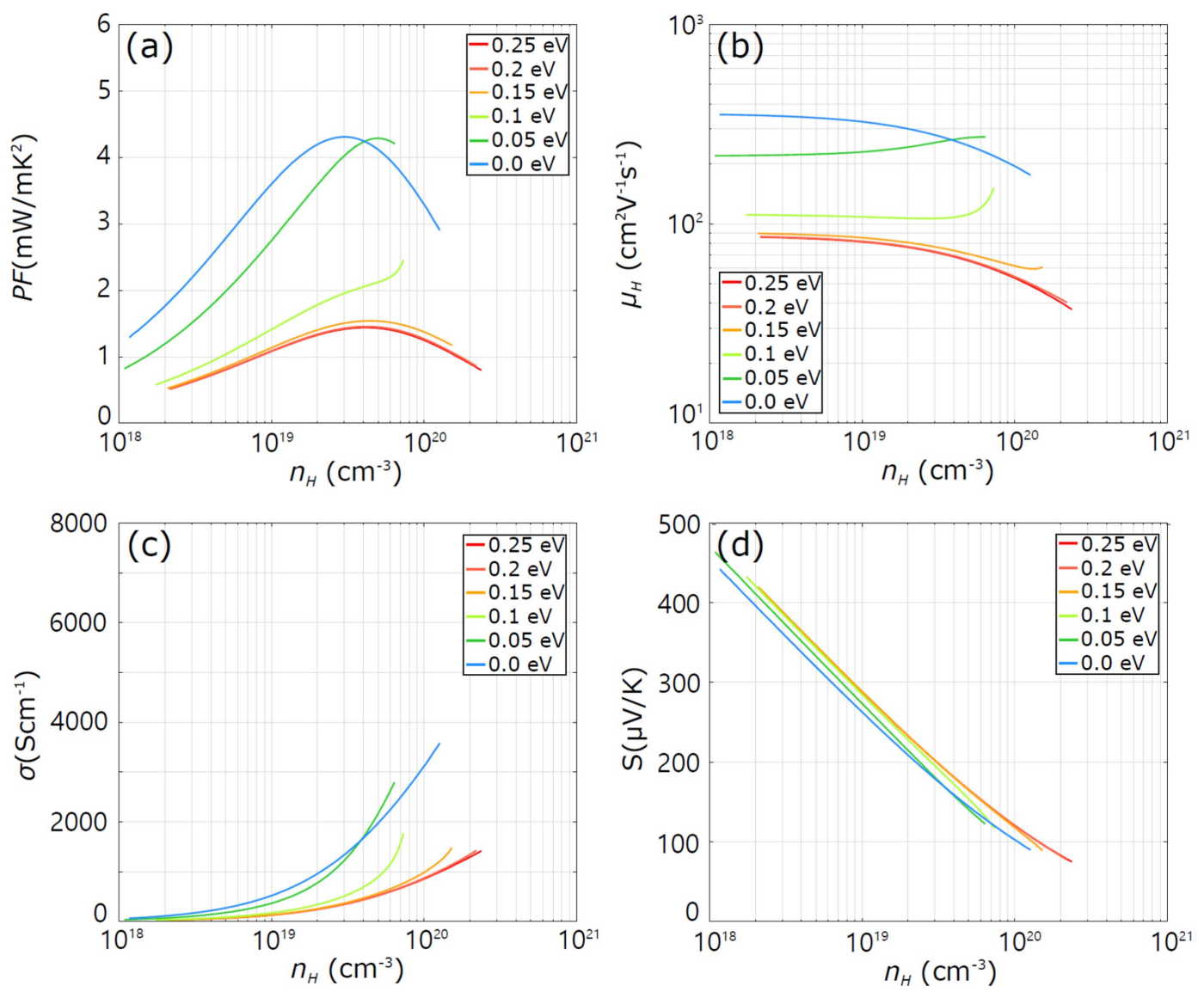

Fig. 4. Calculated transport properties of Case III: (a) power factor, (b) Hall mobility, (c) electrical conductivity, and (d) Seebeck coefficient as a function of Hall carrier concentration. 
calculated combined Hall carrier concentration of the converged case is higher than that of $\Delta E=0.05 \mathrm{eV}$ at all chemical potentials. Since electrical conductivity, Hall mobility, and Hall carrier concentration are interdependent $\left(\sigma=\mu_{H} \times\right.$ $\left.n_{H} \times e\right)$, observed trends in the converged case and for $\Delta E=$ $0.05 \mathrm{eV}$ can be understood as a combined effect among their different chemical potential dependences. Simultaneous improvement in both the Seebeck coefficient and the electrical conductivity of the converged bands results in a substantial increase in its power factor $\left(S^{2} \times \sigma\right)$, as shown in Fig. 2(a).

Apparent decoupling of the Seebeck coefficient and electrical conductivity becomes less obvious once the density-ofstates effective mass $\left(m^{*}\right)$ of the lower valence band $\left(\mathrm{VB}_{2}\right)$ is doubled (Case II in Fig. 1(b)). The power factor of the converged bands (Case II) is still the highest when compared to those of the other bands with $\Delta E>0.0 \mathrm{eV}$. However, the absolute value of the power factor (for Case II: $m^{*}$ of $\mathrm{VB}_{2}=2$ $\times m^{*}$ of $\mathrm{VB}_{1}$ ) is approximately $22 \%$ lower than that computed for Case I $\left(m^{*}\right.$ of $\mathrm{VB}_{2}=m^{*}$ of $\left.\mathrm{VB}_{1}\right)$. We now know that the convergence of bands with the same $m^{*}$ is more beneficial to electrical transport than that of two bands whose values of $m^{*}$ differ by a factor of 2 (assuming that the other band parameters are the same).

The behavior of the Seebeck Pisernko relation for Case II (Fig. 3(d)) is similar to that of Case I (Fig. 2(d)). The converged band ( $0.0 \mathrm{eV}$, blue solid line) has the highest Seebeck coefficient. However, its Hall mobility (Fig. 3(b)) is lower than those of other bands with $\Delta E>0.0 \mathrm{eV}$ at $n_{H}<9 \mathrm{E} 19$ $\left(\mathrm{cm}^{-3}\right)$. This is reasonable as the Hall mobilities of the other bands with $\Delta E>0.0 \mathrm{eV}$ mostly originate from the light $\mathrm{VB}_{1}$ (hence the high Hall mobility) at $n_{H}<9 \mathrm{E} 19\left(\mathrm{~cm}^{-3}\right)$. Similarly, the electrical conductivity of the converged band (0.0 $\mathrm{eV}$, blue solid line) is lowest among all the bands with different $\Delta E$ values. Fortunately, however, the difference in the electrical conductivity is small, which leads to the highest power factor for the converged bands $(0.0 \mathrm{eV}$, blue solid lines in Fig. 3(a)).

Lastly, other two-band model calculations were performed for the case where $m^{*}$ of $\mathrm{VB}_{1}$ is twice as large as that of $\mathrm{VB}_{2}$ (Case III in Fig. 1(c)). The situation of the converged bands in Case III is exactly the same as that in Case II. When $\Delta E$ $>0 \mathrm{eV}$, Cases II and III differ. Since $\mathrm{VB}_{1}$ is heavier than $\mathrm{VB}_{2}$, the total Seebeck coefficient of the converged bands $\left(S_{\text {total }}\right)(0.0 \mathrm{eV}$, blue solid line in Fig. 4(d)) calculated from a conductivity-weighted average of individual Seebeck coefficients ( $S_{\mathrm{VB} 1}$ and $S_{\mathrm{VB} 2}$ from Eq. (6)) can be smaller than the individual Seebeck coefficient of the heavier $\mathrm{VB}_{1}$ (which contributes mainly to transport) (Fig. 4(d)). In a similar vein, the exact opposite reasoning can be applied to explain the highest Hall mobility and electrical conductivity of the converged bands (blue solid line in Fig. 4(b) and 4(c)).

\section{Conclusions}

Although band convergence facilitates higher electrical transport properties, its effect may vary depending on the details of the band parameters of the participating bands. If the band parameters of the converging bands are the same, the decoupling of the Seebeck coefficient and electrical conductivity is the strongest at the highest power factor.

\section{Acknowledgments}

This work was supported by the 2019 Hongik University Research Fund.

\section{REFERENCES}

1. D. M. Rowe, "Applications of Nuclear-Powered Thermoelectric Generators in Space," Appl. Energy, 40 [4] 241-71 (1991).

2. S. I. Kim, K. H. Lee, H. A. Mun, H. S. Kim, S. W. Hwang, J. W. Roh, D. J. Yang, W. H. Shin, X. S. Li, Y. H. Lee, G. J. Snyder, and S. W. Kim, "Dense Dislocation Arrays Embedded in Grain Boundaries for High-Performance Bulk Thermoelectrics," Science, 348 [623] 109-14 (2015).

3. Y. Pei, X. Shi, A. LaLonde, H. Wang, L. Chen, and G. J. Snyder, "Convergence of Electronic Bands for High Performance Bulk Thermoelectrics," Nature, 473 66-9 (2011).

4. H.-S. Kim, N. A. Heinz, Z. M. Gibbs, Y. Tang, S. D. Kang, and G. J. Snyder, "High Thermoelectric Performance in $\left(\mathrm{Bi}_{0.25} \mathrm{Sb}_{0.75}\right)_{2} \mathrm{Te}_{3}$ due to Band Convergence and Improved by Carrier Concentration Control," Mater. Today, 20 [8] 452-59 (2017).

5. A. F. May and G. J. Snyder, "Introduction to Modeling Thermoelectric Transport at High Temperatures", pp. 118 in Materials, Preparation, and Characterization in Thermoelectrics. Ed. by D. M. Rowe, CRC Press, Boca Raton, 2012.

6. Y. I. Ravich, B. A. Efimova, and V. I. Tamarche, "Scattering of Current Carriers and Transport Phenomena in Lead Chalcogenides," Phys. Status Solidi B, 43 [1] 11-33 (1971). 